\title{
Preparation and Capacitive Properties of Polyaniline/Nanocellulose Composites with Core-Shell Structure
}

\author{
Yi Zhou ${ }^{1, a}$, Qinghua Song ${ }^{1, b}$, Banglei Liang ${ }^{2, c}$ and Zongyi Qin ${ }^{2, d}$ \\ ${ }^{1}$ Department of Biological Engineering, Xiangxi National Vocational-Technical College, Jishou, \\ Hunan 416000, China \\ ${ }^{2}$ State Key Laboratory for Modification of Chemical Fibers and Polymer Materials, and College of \\ Material Science and Engineering, Donghua University, Shanghai 201620, China \\ azhouyicynthia@126.com, bsqhsqhsqh123@163.com, ${ }^{\mathrm{c}} 574404270 @ q q . c o m,{ }^{d}$ phqin@dhu.edu.cn
}

Keywords: Polyaniline; Nanocellulose; Nanocomposites; Core-shell structure; Capacitive property Abstract. Polyaniline (PANI) was prepared through in-situ chemical oxidative polymerization of aniline monomers in the presence of nanocelluloses. The as-prepared nanocomposite was characterized by scanning electron microscopy, Fourier transform infrared spectrometry, X-ray diffraction analysis and electrochemical measurements. The results show as-prepared nanocomposite had good core-shell structure. For ammonia gas detection, high sensitivity, relative fast response, short recovery time, and good reproducibility can be achieved for the nanocomposite.

\section{Introduction}

Polyaniline (PANI) has been considered to be the most potential conducting polymers, and has attracted much attention due to their remarkable electrochemical properties, simple and facile synthesis, low cost, good electrical conductivity and good environment stability [1]. Especially, a great enhancement on their electrochemical properties could be achieved when the conductive polymers are in the form of nanostructured materials with highly ordered structure and large specific surface area [1]. However, PANI has poor mechanical properties and processability, and easy to aggregate large particles, which limited its application range. To overcome these disadvantages, conducting polymers could be composited with some nanoscale particles with high mechanical properties [2]. Recently, nanocelluloses have been demonstrated to make up a promising new class of renewable nanomaterials as a support for conducting polymers due to their unique properties including nano-dimensions, high strength and light weight [3]. In this work, nanocelluloses extracted from cotton were used as templates, and nanocellulose/PANI nanocomposites were prepared through in-situ chemical polymerization of aniline monomers in the presence of nanocelluloses. Its potential application as electrode materials for energy storage devices should be evaluated

\section{Experimental}

Materials. Aniline monomers with a molar mass of $93 \mathrm{~g} / \mathrm{mol}$ and a density of $1.02 \mathrm{~g} / \mathrm{cm}^{3}$ were purchased from Pinghu Reagent Co. Ltd. Ammonium peroxydisulfate (APS), hydrochloric acid ( $\mathrm{HCl}$ ) were obtained from Guoyao Group Chemical Reagent Co. Ltd., and used as received without any further purification.

Preparation. The nanocelluloses were extracted from cotton through the hydrolysis process in an APS aqueous solution, and then were well dispersed into $1 \mathrm{M}$ aqueous solution of $\mathrm{HCl}$ [3]. Subsequently, aniline monomers were added into the above suspension under ultrasonic irradiation for $30 \mathrm{~min}$ in an ice bath, and further $1 \mathrm{M} \mathrm{HCl}$ solution containing APS was added dropwise. The weight ratio of aniline monomers to nanocellulose is $3: 1$. After about the reaction of $6 \mathrm{~h}$, the product was washed with deionized water and filtered several times, and then dried under vacuum at $60{ }^{\circ} \mathrm{C}$ for $48 \mathrm{~h}$.

Characterization. The morphologies of nanocomposites were observed on HITACHI S-4800 field emission scanning electron microscopy (FE-SEM). The chemical structures were characterized on a Nicolet NEXUS 670 FTIR spectrometer with a resolution of $4 \mathrm{~cm}^{-1}$ over 32 scans. The crystal 
structures were characterized on a Philips PZ1200 X-ray diffractometer by using $\mathrm{Cu}$ Ka X-rays with a voltage of $40 \mathrm{kV}$ and a current of $30 \mathrm{~mA}$. X-ray diffraction data were collected over an angular range of 5-90 in steps of $0.02^{\circ}$ at room temperature. To study its electrochemical properties, the working electrode containing PANI materials was fabricated by directly pressing as-prepared PANIs onto porous nickel mesh $(0.5 \times 2.0 \mathrm{~cm})$ as the current collector under $5 \mathrm{MPa}$ at $60{ }^{\circ} \mathrm{C}$, and dried under vacuum at room temperature overnight. Cyclic voltammetry $(\mathrm{CV})$ curves was recorded on a $\mathrm{CHI}$ 660D electrochemical working station ( $\mathrm{CH}$ Instrument Company, Shanghai, China) with a three-electrode system (working electrode, a $\mathrm{Pt}$ wire as the counter electrode and $\mathrm{Ag} / \mathrm{AgCl}$ as the reference electrode) in $0.5 \mathrm{M} \mathrm{H}_{2} \mathrm{SO}_{4}+0.5 \mathrm{Na}_{2} \mathrm{SO}_{4}$ electrolyte at room temperature.

\section{Results and Discussion}
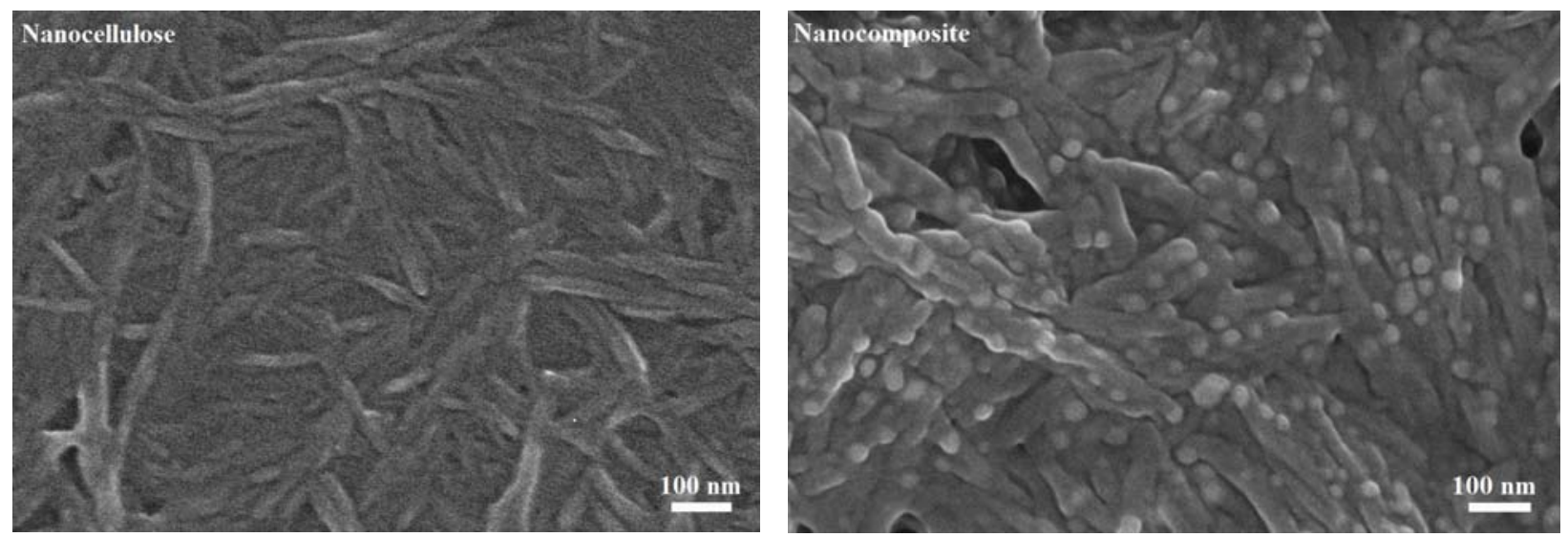

Fig. 1. SEM images for nanocellulose and the resulting nanocomposite.

Morphology. Fig. 1 gives the morphologies of nanocellulose and the resulting nanocomposite. The nanocelluloses extracted from cotton exhibited rod-like morphology with the average diameter of about $30 \mathrm{~nm}$ and length of about $200 \mathrm{~nm}$. It can be found that after the introduction of PANI, the average diameter of the resulting nanocomposite became larger compared with that for the nanocellulose. It shows that polyaniline layer was successfully coated to the surface of nanocelluloses, the nanocomposite exhibited similar rod-like morphology but larger diameter, indicating the formation of core-shell structure.

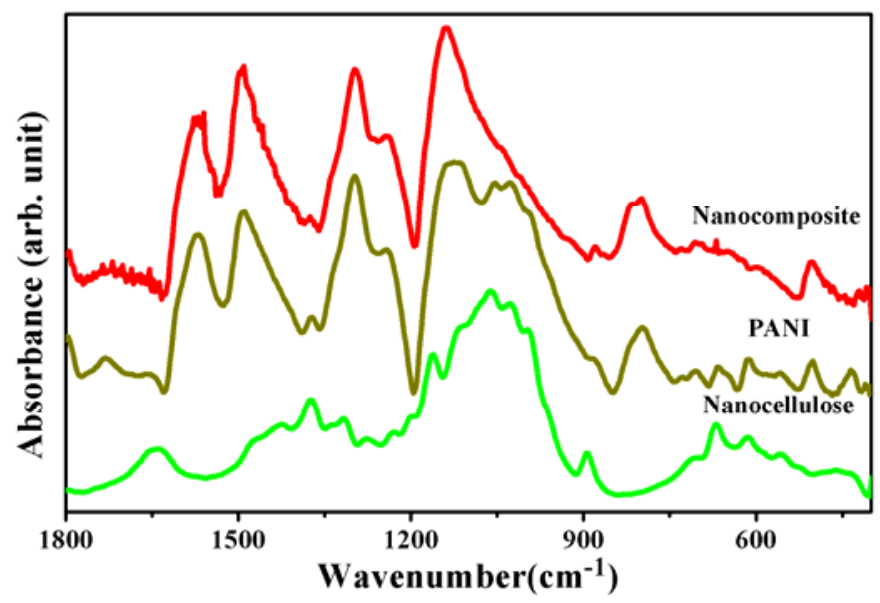

Fig. 2. FT-IR spectra of nanocellulose, PANI and the resulting nanocomposite

Chemical structure. Fig. 2 shows FT-IR spectra of nanocellulose, PANI and the resulting nanocomposite. For nanocellulose, the peak at $1432 \mathrm{~cm}^{-1}$ was assigned to the $\mathrm{H}-\mathrm{C}-\mathrm{H}$ and $\mathrm{O}-\mathrm{C}-\mathrm{H}$ in-plane bending vibrations from various cellulose or lignin components, at $1372 \mathrm{~cm}^{-1}$ to the $\mathrm{C}-\mathrm{H}$ 
deformation vibration, and at 1055 and $1113 \mathrm{~cm}^{-1}$ assigned to the $\mathrm{C}-\mathrm{O}-\mathrm{C}$ stretching of pyranose and glucose ring skeletal vibration, respectively [3]. For PANI, the typical peaks at 1568 and $1490 \mathrm{~cm}^{-1}$ can be assigned to the $\mathrm{C}=\mathrm{C}$ stretching vibration of quinoid and benzenoid rings, respectively, and at 1126 and $1297 \mathrm{~cm}^{-1}$ to in-plane band vibration of $\mathrm{C}-\mathrm{H}$ on the $1,4-$ desubtituted ring, $\mathrm{C}-\mathrm{N}$ stretching vibration mode of the $\mathrm{N}-\mathrm{Ph}-\mathrm{N}$ unit, respectively [1]. All the characteristic peaks of cellulose and PANI can be observed in the infrared spectrum of the nanocomposite. Moreover, the characteristic peaks of cellulose in the spectra of the nanocomposite became weak due to the coverage of polyaniline layer, while some shifting in position for PANI peaks occurred, attributing to the interaction between nanocellulose with PANI chains.

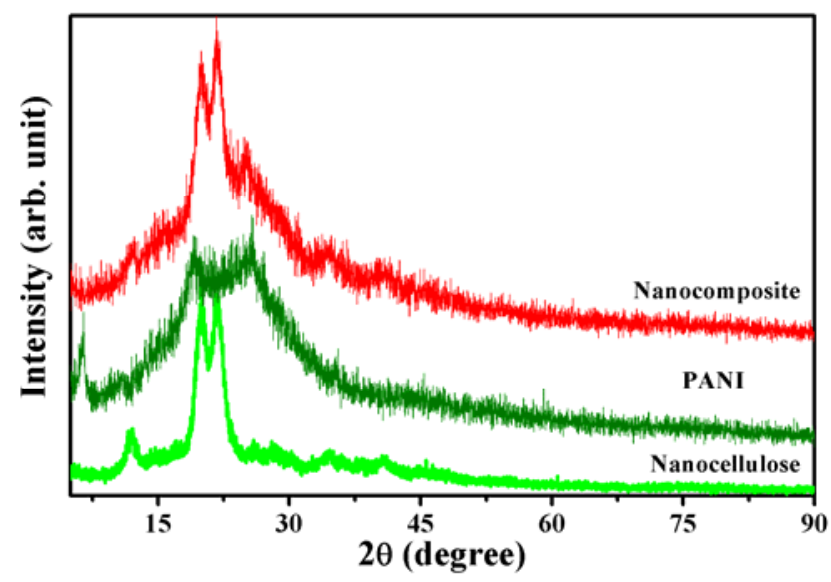

Fig. 3. X-ray diffraction pattern of nanocellulose, PANI and the resulting nanocomposite.

Crystal Structure. As shown in Fig. 3, the diffraction patterns of nanocelluloses exhibited two intense peaks at $2 \theta=20.2^{\circ}$ for the $(110)$ peak and $21.8^{\circ}$ for the (200) peak, and a weak peak at $12.2^{\circ}$ for the (1i10)peak, where $\mathrm{q}$ is the corresponding Bragg angle [3]. Two broad peaks centered at $2 \theta=20^{\circ}$ and $26^{\circ}$ were observed, showing the resulting PANI nanostructures were amorphous. The peak centered at $2 \theta=20^{\circ}$ could be ascribed to periodicity parallel to the polymer chains, while the latter peak may be caused by the periodicity perpendicular to the polymer chains [1]. Moreover, all the characteristic peaks can be found for the nanocomposites, and no obvious change in crystal structure can be observed, implying the ordering of PANI chains did not be improved when nanocelluloses were used as templates.

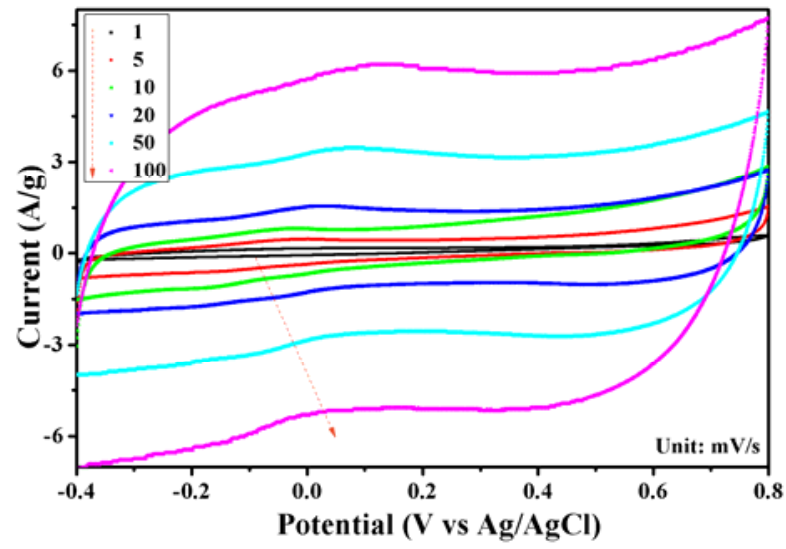

Fig. 4. CV curves of nanocellulose/PANI nanocomposite at various scan rates.

Capacitive property. As illustrated in Fig. 4, the CV curves for the nanocomposite show capacitive-like responses approximately rectangular in shape. Generally, large current and rectangular forms of the voltammogram are believed as the indication of an ideal capacitive nature. The variations of the specific capacitance for the nanocomposites as a function of scan rate are plotted 
in Fig. 4. With the increase of the scan rate, the diffusion rate of electrolyte from solid/liquid interface to electrode materials is not fast enough to satisfy the electrochemical reactions of electrode materials at relatively high scan rate, causing the failure of parts of the electrode materials to complete reaction. Meanwhile, the protons to reach on the surface of the electrode and enter into the active physical phase in a short scan cycle became more impossible at relatively high scan rate, corresponding to the less utilization of active materials. As a result, the specific capacitance decreased obviously. When the scan rate increased from 1 to $100 \mathrm{mV} / \mathrm{s}$, the specific capacitance of the nanocomposite reduced from $161.9,93.2,90.3,84.7,75.6$, and $67.0 \mathrm{~F} / \mathrm{g}$, respectively. This work indicates that the nanocomposite possesses superior electrochemical performance owing to the large electrochemical active area, and high structural stability benefiting from the synergetic effects between nanocellulose and PANI chains.

\section{Conclusions}

The nanocellulose/PANI nanocomposite was prepared by in-situ chemical polymerization of aniline monomers and using CNCs as templates, in which the nanocellulose were extracted from the cotton through hydrolysis process in an APS aqueous solution. SEM observation showed the nanocelluloses exhibited rod-like morphology, and PANI was successfully coated on the surface of the nanocellulose. There is no obvious change in the crystal structure of PANI in the nanocomposite though the existence of interaction between cellulose and PANI chains can be observed in infrared spectrum. Cyclic voltammetry test showed that the relatively high specific capacitance of about $161.9 \mathrm{~F} / \mathrm{g}$ could be achieved for the nanocomposite, benefiting from the large electrochemical active area of PANI, and high structural stability benefiting from the synergetic effects in the nanocomposite.

\section{Acknowledgments}

Financial support from the Fundamental Research Funds for the Central Universities (2232013A3-02) and Innovation Funds for the PhD Students of Donghua University (15D310608) is gratefully acknowledged.

\section{References}

[1] T. Li, Z. Qin, B. Liang, F. Tian, J. Zhao, N. Liu and M. Zhu: Electrochimica Acta Vol. 177 (2015), p. 343.

[2] Y. Xie, Z. Qin and J. Li: Journal of Textile Research Vol. 34 (2013), p. 15.

[3] M. Cheng, Z. Qin, Y. Liu, Y. Qin, T. Li, L. Chen and M. Zhu: Journal of Materials Chemistry A Vol. 2 (2014), p. 251. 